Supporting Information

\title{
Broadband Dielectric Study of Sizable Molecular Glass Formers: Relationship Between Local Structure and Dynamics
}

M. Rams-Baron, ${ }^{1,2, *}$ A. Jedrzejowska, ${ }^{3}$ K. Jurkiewicz, ${ }^{1,2}$ M. Matussek, ${ }^{4}$ K. L. Ngai ${ }^{5}$, M. Paluch ${ }^{1,2}$

${ }^{1}$ August Chetkowski Institute of Physics, University of Silesia, 75 Pulku Piechoty 1, 41-500 Chorzow, Poland

${ }_{2}^{2}$ Silesian Center for Education and Interdisciplinary Research, 75 Pulku Piechoty 1a, 41-500

Chorzow, Poland

${ }^{3}$ PET Diagnostics Department, Maria Sklodowska-Curie Memorial Cancer Centre and Institute of Oncology, Gliwice Branch, Poland

${ }^{4}$ Institute of Chemistry, University of Silesia, Szkolna 9, 40-006 Katowice, Poland

${ }^{5}$ CNR-IPCF, Largo Bruno Pontecorvo 3, I-56127, Pisa, Italy

*Author to whom correspondence should be addressed: marzena.rams-baron@us.edu.pl

\begin{tabular}{|c|c|}
\hline $\mathrm{M}=\mathrm{A}$ & $\mathrm{M}=\mathrm{F}-\left(\mathrm{C}_{8} \mathrm{H}_{17}\right)_{2}$ \\
\hline & \\
\hline $\mathrm{M}=\mathrm{Ph}-\mathrm{OCH}$ & \\
\hline
\end{tabular}




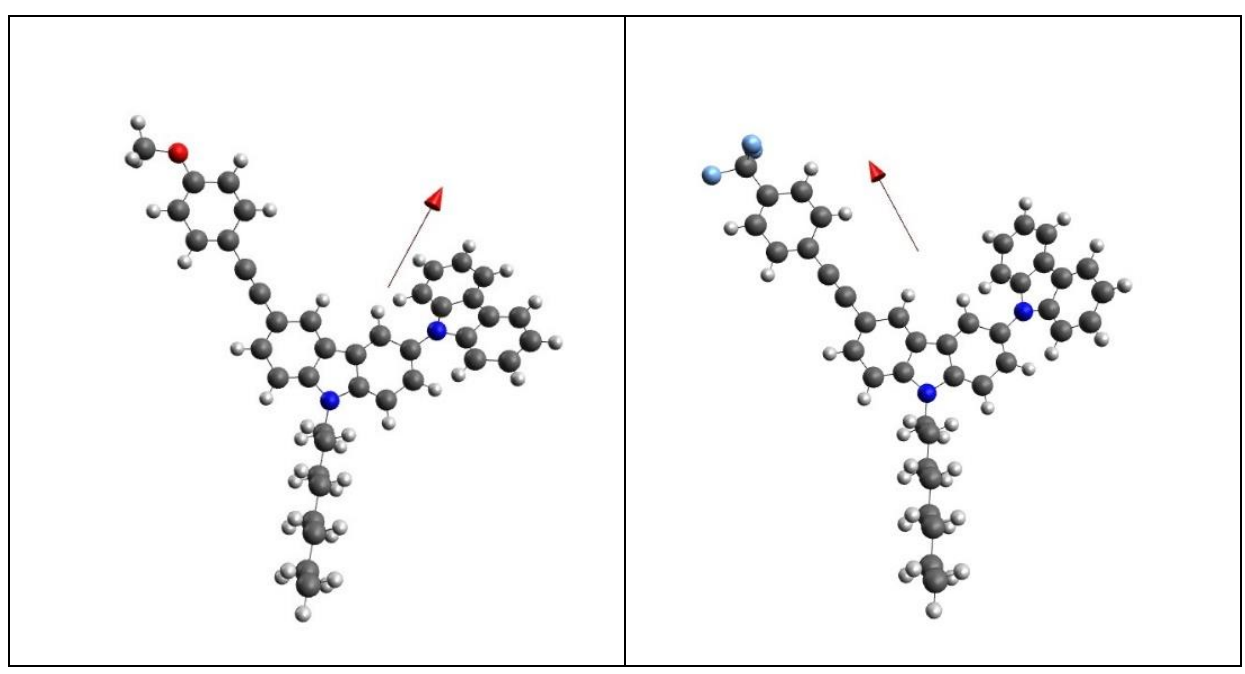

Figure S1. The orientation of molecular (net) dipole moment for the sizable molecules

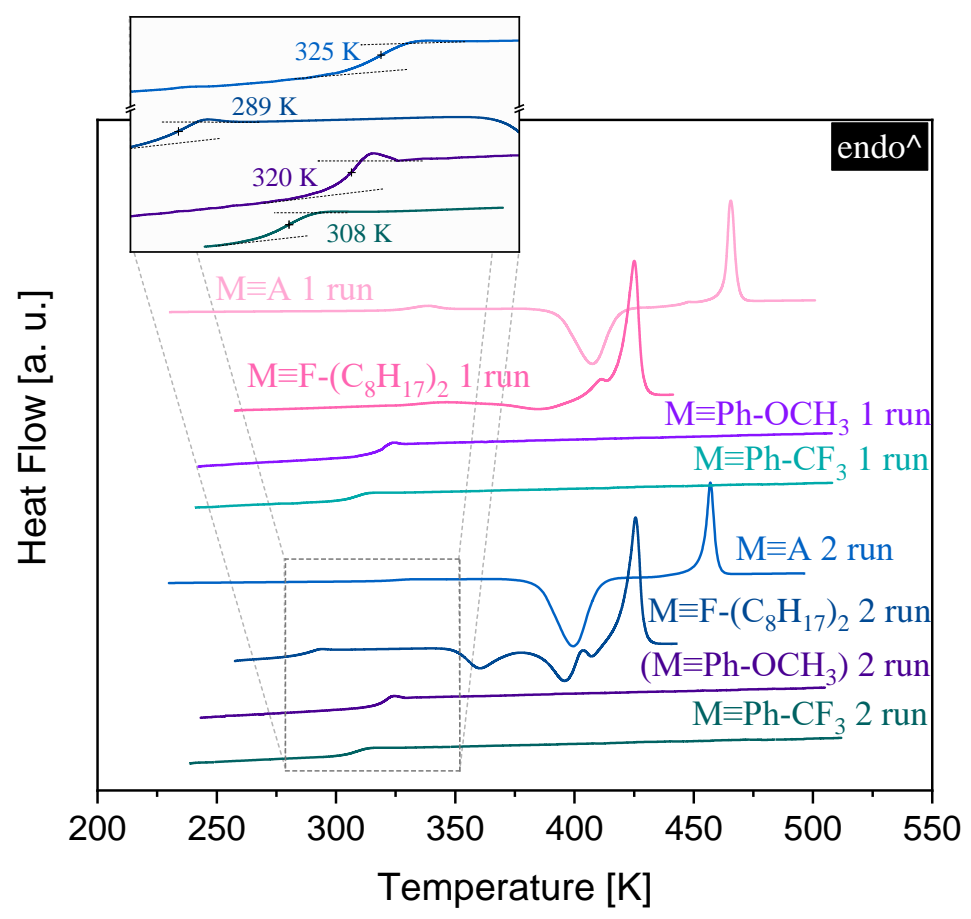

Figure S2. DSC heating scans for investigated sizable systems. 


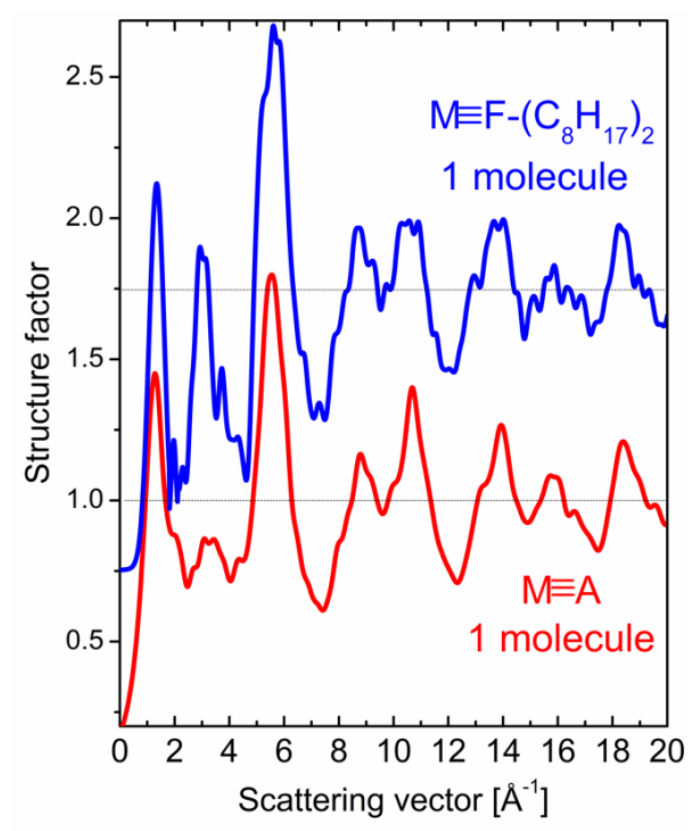

Figure S3. Comparison of X-ray diffraction patterns in the form of structure factors for single molecules of $\mathrm{M} \equiv \mathrm{A}$ and $\mathrm{M} \equiv \mathrm{F}-\left(\mathrm{C}_{8} \mathrm{H}_{17}\right)_{2}$. The data were computed based on the optimized models of single molecules and show that they are similar and do not show pre-peaks in the low scattering vector range where the intermolecular correlations play the main role.

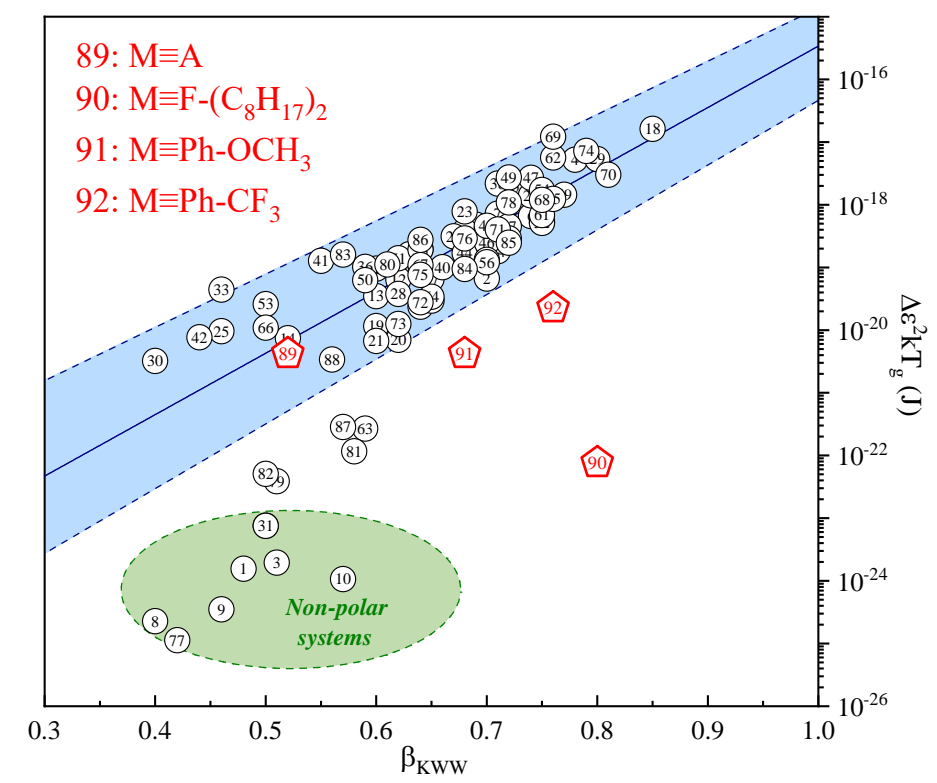

Figure S4. Relationship between the dielectric strength $\Delta \varepsilon$ and the non-exponentiality of structural relaxation (quantified using the $\beta_{\mathrm{KWw}}$ value) presented by depicted $\Delta \varepsilon^{2} k T_{g}$ against $\beta_{\mathrm{KWw}}$ according to Paluch et al. The values $1-88$ were taken together with the numbers explanation from Phys. Rev. Lett. 116, 025702 (2016). Reprinted with permission from M. Paluch, J. Knapik, Z. Wojnarowska, A. Grzybowski, K. L. Ngai, Phys. Rev. Lett. 116, 025702 (2016) Copyright 2016 by the American Physical Society. The numbers 89 - 92 correspond to 
investigated sizable glass-formers i.e. 89: $\mathrm{M} \equiv \mathrm{A}, 90: \mathrm{M} \equiv \mathrm{F}-\left(\mathrm{C}_{8} \mathrm{H}_{17}\right)_{2}, 91: \mathrm{M} \equiv \mathrm{Ph}-\mathrm{OCH}_{3}, 92$ : $\mathrm{M} \equiv \mathrm{Ph}-\mathrm{CF}_{3}$.

Table S1. The values of the diffraction peak maximum positions and full widths at the half maximum for the three pseudo-Voigt profiles fitted to the diffraction data in the range up to $1.75 \AA^{-1}$.

\begin{tabular}{|c|c|c|c|c|c|c|}
\hline \multirow[b]{2}{*}{ Compound } & \multicolumn{6}{|c|}{ Fitting parameters $\left[\AA^{-1}\right]$} \\
\hline & $Q_{M P}$ & fwhm ${ }_{M P}$ & $Q_{P P 1}$ & fwhmpP1 & $Q_{P P 2}$ & fwhm $_{\mathrm{PP} 2}$ \\
\hline $\mathbf{M} \equiv \mathbf{A}$ & $\begin{array}{c}1.510 \pm \\
0.001\end{array}$ & $\begin{array}{c}0.367 \pm \\
0.002\end{array}$ & $\begin{array}{c}0.407 \pm \\
0.003\end{array}$ & $\begin{array}{c}0.265 \pm \\
0.013\end{array}$ & $\begin{array}{c}0.847 \pm \\
0.005\end{array}$ & $\begin{array}{c}0.280 \pm \\
0.015\end{array}$ \\
\hline$M \equiv F-\left(C_{8} H_{17}\right)_{2}$ & $\begin{array}{c}1.459 \pm \\
0.001\end{array}$ & $\begin{array}{c}0.224 \pm \\
0.00159\end{array}$ & $\begin{array}{c}0.428 \pm \\
0.002\end{array}$ & $\begin{array}{c}0.216 \pm \\
0.007\end{array}$ & $\begin{array}{c}1.070 \pm \\
0.005\end{array}$ & $\begin{array}{c}0.325 \pm \\
0.014\end{array}$ \\
\hline $\mathrm{M} \equiv \mathrm{Ph}-\mathrm{OCH}_{3}$ & $\begin{array}{c}1.485 \pm \\
0.001\end{array}$ & $\begin{array}{c}0.281 \pm \\
0.004\end{array}$ & $\begin{array}{c}0.426 \pm \\
0.004\end{array}$ & $\begin{array}{c}0.241 \pm \\
0.015\end{array}$ & $\begin{array}{c}1.120 \pm \\
0.013\end{array}$ & $\begin{array}{c}0.510 \pm \\
0.013\end{array}$ \\
\hline $\mathbf{M} \equiv \mathbf{P h}-\mathbf{C F}_{\mathbf{3}}$ & $\begin{array}{c}1.476 \pm \\
0.001\end{array}$ & $\begin{array}{c}0.257 \pm \\
0.003\end{array}$ & $\begin{array}{c}0.356 \pm \\
0.004\end{array}$ & $\begin{array}{c}0.189 \pm \\
0.015\end{array}$ & $\begin{array}{c}1.148 \pm \\
0.016\end{array}$ & $\begin{array}{c}0.523 \pm \\
0.015\end{array}$ \\
\hline
\end{tabular}

\section{Experimental details}

\section{Synthesis}

\section{Materials}

All reagents were purchased from TCI Chemicals or Sigma-Aldrich and were used without further purification. Solvents were distilled by using standard methods and purged with argon before use. Column chromatography was carried out on Merck silica gel (230 - 400 mesh). Thin-layer chromatography (TLC) was performed on silica gel (Merck TLC Silica Gel 60, F254). NMR spectra were taken on Bruker Avance 400 (400 MHz) spectrometer. Chemical shifts $(\delta)$ are reported in parts per million $(\mathrm{ppm})$ relative to traces of $\mathrm{CHCl}_{3}\left(\delta_{\mathrm{H}}=7.26 \mathrm{ppm}, \delta_{\mathrm{C}}\right.$ $=77.0 \mathrm{ppm}$ ).

a) Synthesis of intermediates 1-7
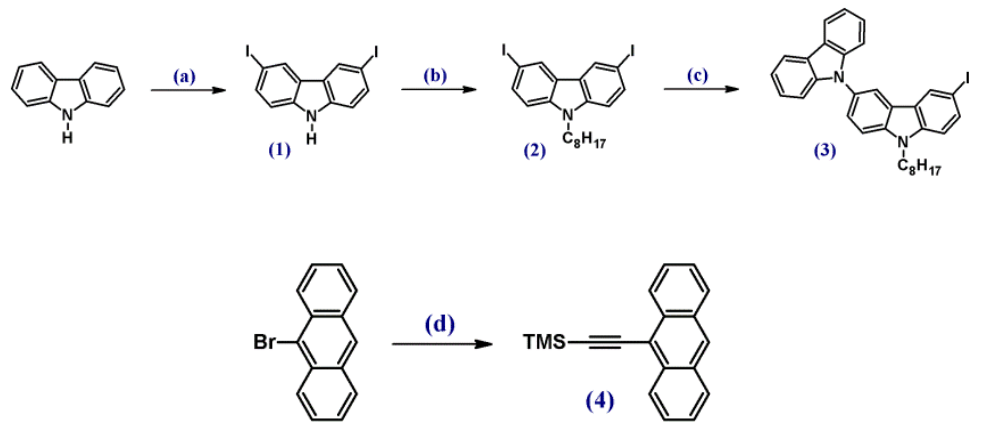


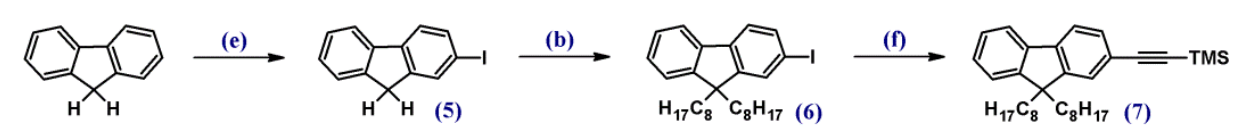

Synthesis of compounds 1-7. Reagents and conditions: (a) $\mathrm{KI}, \mathrm{KIO}_{3}, \mathrm{AcOH}$, $\mathrm{rfx}, 15$ min.; (b) $50 \% \mathrm{NaOH}$, TBAB, $\mathrm{n}-\mathrm{C}_{8} \mathrm{H}_{17}$, DMSO, r.t., 12h; (c) carbazole, CuI, 1,10-phenantroline, $\mathrm{K}_{2} \mathrm{CO}_{3}$, DMF, rfx, 30h; (d) TMSA (1.4 eq), $\mathrm{Pd}\left(\mathrm{PPh}_{3}\right)_{4}$, CuI, PhMe/TEA, $100{ }^{\circ} \mathrm{C}, 24 \mathrm{~h}$; (e) $\mathrm{I}_{2}, \mathrm{H}_{5} \mathrm{IO}_{6}$, $\mathrm{AcOH} / \mathrm{H}_{2} \mathrm{O} / \mathrm{H}_{2} \mathrm{SO}_{4}, 70{ }^{\circ} \mathrm{C}$, 6h. (f) TMSA (1.2 eq), $\mathrm{Pd}\left(\mathrm{PPh}_{3}\right)_{4}, \mathrm{CuI}$, TEA, r.t., 24h.

b) Synthesis of target molecules $\mathrm{M} \equiv \mathrm{A}, \mathrm{M} \equiv \mathrm{F}-\left(\mathrm{C}_{8} \mathrm{H}_{17}\right)_{2}, \mathrm{M} \equiv \mathrm{Ph}-\mathrm{OCH}_{3}$ and $\mathrm{M} \equiv \mathrm{Ph}-\mathrm{CF}_{3}$

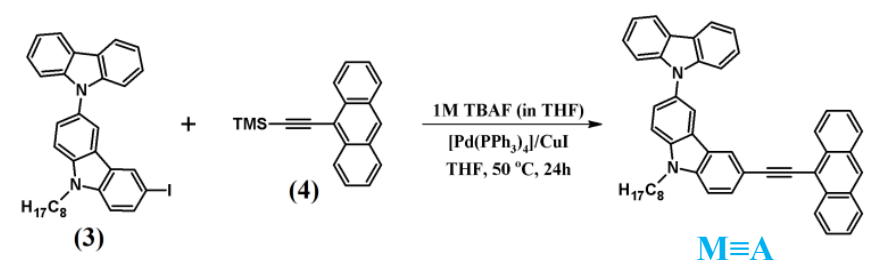

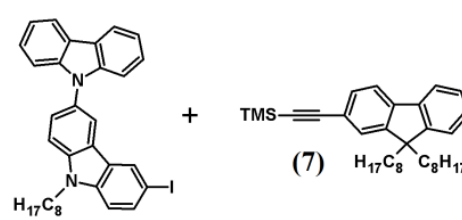

(3)

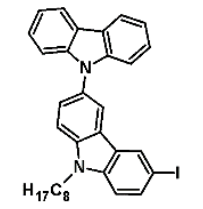

(3)

$\mathrm{R}=\mathrm{OCH}_{3}$ or $\mathrm{CF}_{3}$

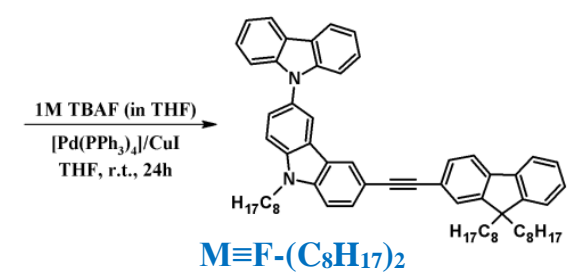

$\mathrm{M} \equiv \mathrm{F}-\left(\mathrm{C}_{8} \mathrm{H}_{17}\right)_{2}$
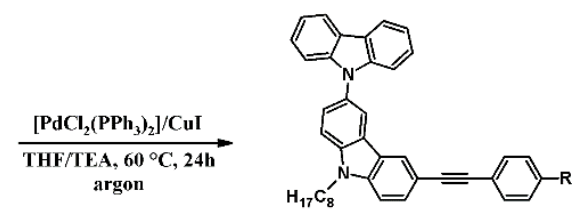

$\mathrm{R}=\mathrm{OCH}_{3}$ or $\mathrm{CF}_{3}$

$\mathrm{M} \equiv \mathrm{Ph}-\mathrm{OCH}_{3} \quad \mathrm{M} \equiv \mathrm{Ph}-\mathrm{CF}_{3}$

The synthetic route for the preparation of compounds $\mathrm{M} \equiv \mathrm{A}, \mathrm{M} \equiv \mathrm{F}-\left(\mathrm{C}_{8} \mathrm{H}_{17}\right)_{2}, \mathrm{M} \equiv \mathrm{Ph}-\mathrm{OCH}_{3}$ and $\mathrm{M} \equiv \mathrm{Ph}-\mathrm{CF}_{3}$.

\section{3,6-diiodocarbazole (1)}

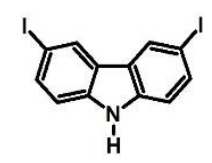

Carbazole (20 g, $119.6 \mathrm{mmol})$ was dissolved in boiling glacial acetic acid (350 mL) under argon atmosphere, and then potassium iodide $(26.5 \mathrm{~g}, 159.6 \mathrm{mmol})$ was added. The solution was cooled, and ground potassium iodate $(38.5 \mathrm{~g}, 179.9 \mathrm{mmol})$ was slowly added in a small portion. The resulting mixture was heated under reflux until it acquired a clear straw-colored tint (15 
min). After this time, the hot solution was decanted from the undissolved potassium iodate, and was cooled to $45{ }^{\circ} \mathrm{C}$. The resulting precipitate was filtered, and the crude product was recrystallized from methanol $(100 \mathrm{~mL})$ to afford a white solid $(26.1 \mathrm{~g}, 52 \%$ yield $) .{ }^{1} \mathbf{H}$ NMR $\left(400 \mathrm{MHz}, \mathrm{CDCl}_{3}\right): \delta 8.32(\mathrm{~s}, 2 \mathrm{H}), 8.07($ br s, $1 \mathrm{H}), 7.68\left(\mathrm{dd}, J_{1}=8.5, J_{2}=1.2 \mathrm{~Hz}, 2 \mathrm{H}\right), 7.21(\mathrm{~d}$, $J=8.5 \mathrm{~Hz}, 2 \mathrm{H}) .{ }^{13} \mathbf{C}$ NMR $\left(101 \mathrm{MHz}, \mathrm{CDCl}_{3}\right): \delta 138.42,134.76,129.21,123.44,112.60,82.48$.

\section{3,6-diiodo-N-octylcarbazole (2)}

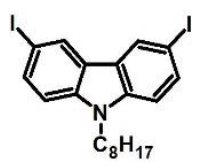

To a solution of $1(15 \mathrm{~g}, 35.8 \mathrm{mmol})$ in DMSO (400 mL) were added a catalytic amount of tetrabutylammonium bromide $(0.8 \mathrm{~g}, 2.5 \mathrm{mmol})$, and $50 \%$ aqueous $\mathrm{NaOH}(22 \mathrm{~mL})$. The reaction mixture was vigorously stirred for $5 \mathrm{~min}$., and 1-bromooctane $(7.4 \mathrm{~mL}, 43.0 \mathrm{mmol})$ was added. The resulting mixture was stirred at room temperature for $12 \mathrm{~h}$. After this time, the mixture was extracted with ethyl acetate. The combined organic layers were dried under anhydrous $\mathrm{MgSO}_{4}$ and filtered. After removing the solvent under reduced pressure, the crude product was purified by flash column chromatography (silica gel, hexane) afforded a white solid (16.9 g, 89\% yield). ${ }^{1} \mathbf{H}$ NMR (400 MHz, $\left.\mathrm{CDCl}_{3}\right): \delta 8.32(\mathrm{~d}, J=1.6,2 \mathrm{H}), 7.71\left(\mathrm{dd}, J_{l}=\right.$ $\left.8.6 \mathrm{~Hz}, J_{2}=1.5 \mathrm{~Hz}, 2 \mathrm{H}\right), 7.16(\mathrm{~d}, J=8.6 \mathrm{~Hz}, 2 \mathrm{H}), 4.21(\mathrm{t}, J=7.1 \mathrm{~Hz}, 2 \mathrm{H}), 1.84-1.75(\mathrm{~m}, 2 \mathrm{H})$, $1.33-1.18(\mathrm{~m}, 10 \mathrm{H}), 0.85(\mathrm{t}, J=7.1 \mathrm{~Hz}, 3 \mathrm{H}) .{ }^{13} \mathbf{C ~ N M R}\left(101 \mathrm{MHz}, \mathrm{CDCl}_{3}\right): \delta 139.50,134.50$, 129.36, 123.97, 110.89, 81.60, 43.26, 31.77, 29.30, 29.12, 28.83, 27.21, 22.58, 14.02.

3-iodo-6-(9H-carbazol-9-yl)-9-octylcarbazole (3)

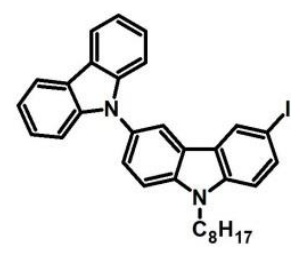

A mixture of 2 (10.6 g, $20 \mathrm{mmol})$, carbazole (3 g, $18 \mathrm{mmol}), 1,10$-phenantroline (540 mg, 3 $\mathrm{mmol}), \mathrm{K}_{2} \mathrm{CO}_{3}(3.8 \mathrm{~g}, 28.6 \mathrm{mmol})$, and $\mathrm{CuI}(286 \mathrm{mg}, 1.5 \mathrm{mmol})$ in $25 \mathrm{~mL}$ of anhydrous DMF was heated under reflux for $30 \mathrm{~h}$ under argon atmosphere. After this time, the mixture was cooled, diluted with $\mathrm{CH}_{2} \mathrm{Cl}_{2}$, and filtered. The filtrate was concentrated under reduced pressure. The crude product was purified by column chromatography (silica gel, hexane/dichloromethane, 10:1 $\rightarrow$ 5:1 v/v) to give 3 as a white solid (6.1 g, $60 \%$ yield). ${ }^{1} \mathbf{H}$ NMR $\left(400 \mathrm{MHz}, \mathrm{CDCl}_{3}\right): \delta 8.37(\mathrm{~d}, J=1.6 \mathrm{~Hz}, 1 \mathrm{H}), 8.21-8.15(\mathrm{~m}, 3 \mathrm{H}), 7.76\left(\mathrm{dd}, J_{1}=8.6 \mathrm{~Hz}, J_{2}=\right.$ 
1.7 Hz, 1H), 7.65-7.55 (m, 2H), 7.45-7.34 (m, 4H), 7.33-7.26 (m, 3H), 4.36 (t, J= 7.3 Hz, 2H), 2.03-1.86 (m, 2H), 1.47-1.18 (m, 10H), $0.88(\mathrm{t}, J=6.9 \mathrm{~Hz}, 3 \mathrm{H}) .{ }^{13} \mathbf{C}$ NMR $\left(101 \mathrm{MHz}, \mathrm{CDCl}_{3}\right)$ : $\delta 141.90,140.29,139.50,134.57,129.55,129.42,125.94,125.68,125.04,123.21,122.50$, $120.38,119.72$, 119.66, 111.16, 109.97, 109.84, 81.67, 43.26, 31.78, 29.30, 29.11, 28.82, 27.21, $22.60,14.01$.

9-(trimethylsilylethynyl)anthracene (4)

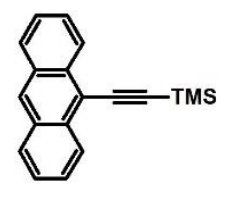

To a stirred solution of 9-bromoanthracene (4 g, $15.6 \mathrm{mmol}$ ) in $150 \mathrm{~mL}$ degassed $\mathrm{PhMe} / \mathrm{TEA}$ $(2: 1 \mathrm{v} / \mathrm{v})$ was added a catalytic mixture of $\mathrm{CuI}(297 \mathrm{mg}, 1.56 \mathrm{mmol})$ and $\mathrm{Pd}\left(\mathrm{PPh}_{3}\right)_{4}(901 \mathrm{mg}$, $0.8 \mathrm{mmol}$ ). The mixture was stirred under argon for $15 \mathrm{~min}$. After this time, trimethylsilylacetylene $(3.1 \mathrm{~mL}, 21.8 \mathrm{mmol}, 1.4 \mathrm{eq})$ was injected through the septum, and the mixture was heated at $100{ }^{\circ} \mathrm{C}$ for additional $24 \mathrm{~h}$ (the completion of the reaction was monitored by TLC). The reaction mixture was filtered and filtrate was evaporated under reduced pressure. After removal of the solvent, the residue was purified by flash column chromatography (silica gel, hexane) to afford a dark red solid (3.3 g, 78\% yield). ${ }^{1} \mathbf{H}$ NMR $\left(400 \mathrm{MHz}, \mathrm{CDCl}_{3}\right): \delta 8.57$ $8.53(\mathrm{~m}, 2 \mathrm{H}), 8.42(\mathrm{~s}, 1 \mathrm{H}), 8.00(\mathrm{~d}, J=8.4 \mathrm{~Hz}, 2 \mathrm{H}), 7.61-7.55(\mathrm{~m}, 2 \mathrm{H}), 7.53-7.47(\mathrm{~m}, 2 \mathrm{H}), 0.42$ (s, 9H). ${ }^{13} \mathbf{C}$ NMR (101 MHz, $\left.\mathrm{CDCl}_{3}\right): \delta 133.04,131.17,128.78,128.05,126.88,126.82$, $125.73,117.23,106.29,101.88,0.50$.

2-iodofluorene (5)

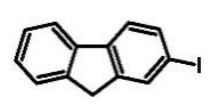

Fluorene (30 g, $180.4 \mathrm{mmol})$ was dissolved in $300 \mathrm{~mL}$ of the boiling mixture of acetic acid, water, and sulphuric acid (100:20:3 v/v). The mixture was cooled slowly to $70{ }^{\circ} \mathrm{C}$, and periodic acid dihydrate $(6.9 \mathrm{~g}, 30.25 \mathrm{mmol})$ and powdered iodine $(15.3 \mathrm{~g}, 60.25 \mathrm{mmol})$ were added. The resulting mixture was stirred for $6 \mathrm{~h}$ (yellow precipitate was formed). After being cooled to room temperature, the yellow solid was collected by filtration, washed with saturated $\mathrm{NaHCO}_{3}$ and water. The crude product was recrystallized from methanol $(1 \times 150 \mathrm{~mL})$ and hexane $(1 \times$ $150 \mathrm{~mL})$ to give a pale yellow solid $(29.5 \mathrm{~g}, 54 \%$ yield $) .{ }^{1} \mathbf{H} \mathbf{~ N M R}\left(400 \mathrm{MHz}, \mathrm{CDCl}_{3}\right): \delta 7.92$ (s, 1H), 7.79 (d, $J=7.4 \mathrm{~Hz}, 1 \mathrm{H}), 7.72(\mathrm{~d}, J=8.0 \mathrm{~Hz}, 1 \mathrm{H}), 7.56(\mathrm{~d}, J=7.8 \mathrm{~Hz}, 2 \mathrm{H}), 7.42-7.34$ 
(m, 2H), 3.90 (s, 2H). ${ }^{13}$ C NMR (101 MHz, $\left.\mathrm{CDCl}_{3}\right): \delta$ 145.52, 142.73, 141.31, 140.80, 135.88, $134.24,127.33,127.01,125.07,121.53,120.05,91.85,36.64$.

2-iodo-9,9-dioctylfluorene (6)

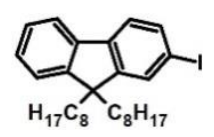

To a solution of $5(15 \mathrm{~g}, 51.3 \mathrm{mmol})$ in DMSO $(500 \mathrm{~mL})$ a catalytic amount of tetrabutylammonium bromide $(0.8 \mathrm{~g}, 2.6 \mathrm{mmol})$ and $50 \%$ aqueous $\mathrm{NaOH}$ solution $(20 \mathrm{~mL})$ were added. When the reaction mixture turned deep red, 1-bromooctane (19.5 mL, $112.8 \mathrm{mmol})$ was added and the resulting mixture was vigorously stirred at room temperature for $12 \mathrm{~h}$. After this time, the mixture was extracted with ethyl acetate. The combined organic layers were dried over anhydrous $\mathrm{MgSO}_{4}$ and filtered. After removing the solvent under reduced pressure, the crude product was purified by flash column chromatography (silica gel, hexane) to afford a pale-yellow viscous oil (20.9 g, 79\% yield). ${ }^{1} \mathbf{H}$ NMR (400 MHz, $\left.\mathrm{CDCl}_{3}\right): \delta$ 7.69-7.66 (m, 3H), $7.46(\mathrm{~d}, J=8.3 \mathrm{~Hz}, 1 \mathrm{H}), 7.36-7.34(\mathrm{~m}, 3 \mathrm{H}), 1.97-1.91$ (m, 4H), 1.26-1.07 (m, 20H), 0.85 (t, $J$ $=7.1 \mathrm{~Hz}, 6 \mathrm{H}), 0.67-0.58(\mathrm{~m}, 4 \mathrm{H}) .{ }^{13} \mathbf{C} \mathbf{N M R}\left(101 \mathrm{MHz}, \mathrm{CDCl}_{3}\right): \delta 153.22,150.13,140.86$, $140.11,135.86,132.15,127.71,126.97,122.98,121.49,119.84,92.55,55.36,40.33,31.87$, $30.01,29.2,29.21,23.76,22.71,14.16$.

2-(trimethylsilylethynyl)-9,9-dioctylfluorene (7)

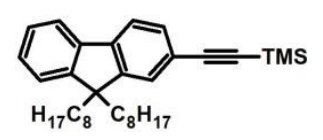

To a solution of $6(10 \mathrm{~g}, 19.36 \mathrm{mmol})$ in $150 \mathrm{~mL}$ degassed TEA a catalytic mixture of $\mathrm{Pd}\left(\mathrm{PPh}_{3}\right)_{4}$ (1.12 g, $0.97 \mathrm{mmol}, 5 \mathrm{~mol}-\%)$ and CuI (184 mg, $0.97 \mathrm{mmol}, 5 \mathrm{~mol}-\%)$ was added. The mixture was stirred under argon for $20 \mathrm{~min}$, then trimethylsilylacetylene $(3.26 \mathrm{~mL}, 23.2 \mathrm{mmol})$ was injected through the septum and the mixture was stirred at room temperature for $24 \mathrm{~h}$ (the completion of the reaction was monitored by silica TLC). The reaction mixture was filtered and the filtrate was evaporated under reduced pressure. After removal of the solvent, the residue was purified by column chromatography (silica gel, hexane) to give $\mathbf{7}$ as a pale-yellow viscous oil (6.4 g, 68\% yield). ${ }^{1} \mathbf{H}$ NMR (400 MHz, $\left.\mathrm{CDCl}_{3}\right): \delta 7.69-7.65(\mathrm{~m}, 1 \mathrm{H}), 7.61(\mathrm{~d}, J=7.8 \mathrm{~Hz}$, $1 \mathrm{H}), 7.47-7.41(\mathrm{~m}, 2 \mathrm{H}), 7.35-7.29(\mathrm{~m}, 3 \mathrm{H}), 2.00-1.86(\mathrm{~m}, 4 \mathrm{H}), 1.28-0.97(\mathrm{~m}, 20 \mathrm{H}), 0.82(\mathrm{t}, J=$ $7.1 \mathrm{~Hz}, 6 \mathrm{H}), 0.67-0.48(\mathrm{~m}, 4 \mathrm{H}), 0.28(\mathrm{~s}, 9 \mathrm{H}) .{ }^{13} \mathbf{C}$ NMR (101 MHz, acetone-d $\left.\mathrm{d}_{6}\right): \delta 150.85$, 
$150.67,141.90,140.35,130.96,127.77,127.05,126.04,122.90,121.49,120.15,119.72$, $106.18,93.35,55.08,39.94,31.61,29.68,29.05,29.00,23.58,22.38,13.49,0.72$.

General Sonogashira cross-coupling procedure for the preparation of target compounds

a) $\mathrm{M} \equiv \mathrm{A}$ and $\mathrm{M} \equiv \mathrm{F}-\left(\mathrm{C}_{8} \mathrm{H}_{17}\right)_{2}$

A solution of 3 ( $2 \mathrm{~g}, 3.5 \mathrm{mmol})$ in $40 \mathrm{~mL}$ THF was bubbled with argon and stirred for 10

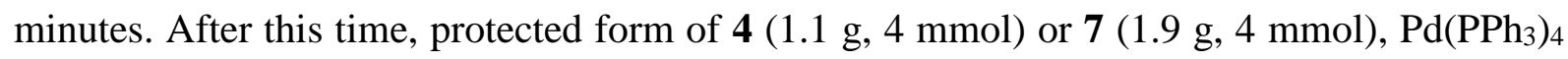
(202 mg, $0.18 \mathrm{mmol}, 5 \%$-mol), and $\mathrm{CuI} \mathrm{(33} \mathrm{mg,} 0.18 \mathrm{mmol}, 5 \%$-mol) were added, and the resulting mixture was bubbled with argon for another 10 minutes. Then, TBAF (4.5 mL, 1M in THF, $4.5 \mathrm{mmol}$ ) was injected through the septum, and the mixture was stirred at room temperature (in the case of $\mathbf{M} \equiv \mathbf{F}-\left(\mathbf{C}_{\mathbf{8}} \mathbf{H}_{17}\right)_{2}$ ) or heated at $50{ }^{\circ} \mathrm{C}$ (in the case of $\mathbf{M} \equiv \mathbf{A}$ ) for $24 \mathrm{~h}$. After this time, the reaction mixture was filtered and the filtrate was evaporated under reduced pressure. The residue was purified by column chromatography.

\section{b) $\mathrm{M} \equiv \mathrm{Ph}-\mathrm{OCH}_{3}$ and $\mathrm{M} \equiv \mathrm{Ph}-\mathrm{CF}_{3}$}

A solution of compound 3 (2 g, $3.5 \mathrm{mmol})$ in $80 \mathrm{~mL}$ degassed THF and TEA (3:1 v/v) was bubbled with argon and stirred for 10 minutes. After this time, $\mathrm{PdCl}_{2}\left(\mathrm{PPh}_{3}\right)_{4}(196 \mathrm{mg}, 0.28$ mmol, 8\%-mol) and $\mathrm{CuI}(53 \mathrm{mg}, 0.28 \mathrm{mmol}, 8 \%$-mol) were added, and the resulting mixture was bubbled with argon for another 10 minutes. 4-ethynyl- $\alpha, \alpha, \alpha$-trifluorotoluene (in the case of $\left.\mathbf{M} \equiv \mathbf{P h}-\mathbf{C F}_{3}\right)(715 \mathrm{mg}, 4.2 \mathrm{mmol}, 1.2 \mathrm{eq})$ or 4-ethynylanisole (in the case of $\mathbf{M} \equiv \mathbf{P h}-\mathbf{O C H}$ ) (555 $\mathrm{mg}, 4.2 \mathrm{mmol}, 1.2 \mathrm{eq}$ ) in $10 \mathrm{~mL}$ THF was then injected through the septum, and the mixture was stirred at $60{ }^{\circ} \mathrm{C}$ for $24 \mathrm{~h}$ under argon atmosphere. After this time, the reaction mixture was filtered and the filtrate was evaporated under reduced pressure. The residue was purified by column chromatography.

\section{9-((6-(9H-carbazol-9-yl)-9-octylcarbazol-3-yl)ethynyl)anthracene $(\mathrm{M} \equiv \mathrm{A})$}

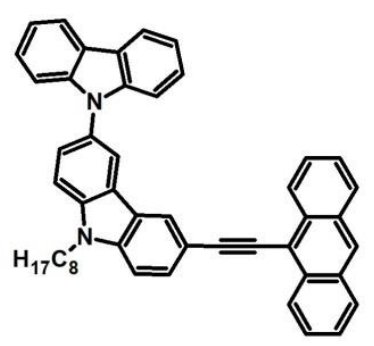

The crude product was purified by column chromatography (silica gel, hexane/dichloromethane, 5:1 $\rightarrow 5: 3 \mathrm{v} / \mathrm{v}$ ) and recrystallized from pentane, to give $\mathbf{M} \equiv \mathbf{A}$ as a 
yellow solid (1.3 g, 58\% yield). ${ }^{1} \mathbf{H}$ NMR (400 MHz, $\left.\mathrm{CDCl}_{3}\right): \delta 8.75(\mathrm{~d}, J=8.7 \mathrm{~Hz}, 2 \mathrm{H}), 8.51$ $(\mathrm{d}, J=1.3 \mathrm{~Hz}, 1 \mathrm{H}), 8.41(\mathrm{~s}, 1 \mathrm{H}), 8.35(\mathrm{~d}, J=1.8 \mathrm{~Hz}, 1 \mathrm{H}), 8.22(\mathrm{~d}, J=7.8 \mathrm{~Hz}, 2 \mathrm{H}), 8.02(\mathrm{~d}, J=$ $8.4 \mathrm{~Hz}, 2 \mathrm{H}), 7.94\left(\mathrm{dd}, J_{l}=8.5 \mathrm{~Hz}, J_{2}=1.5 \mathrm{~Hz}, 1 \mathrm{H}\right), 7.68-7.59(\mathrm{~m}, 4 \mathrm{H}), 7.53-7.50(\mathrm{~m}, 3 \mathrm{H})$, 7.46-7.45 (m, 4H), 7.35-7.31 (m, 2H), $4.41(\mathrm{t}, J=7.2 \mathrm{~Hz}, 2 \mathrm{H}), 2.03-1.96(\mathrm{~m}, 2 \mathrm{H}), 1.52-1.25$ $(\mathrm{m}, 10 \mathrm{H}), 0.92(\mathrm{t}, J=6.9 \mathrm{~Hz}, 3 \mathrm{H}) .{ }^{13} \mathbf{C} \mathbf{N M R}\left(101 \mathrm{MHz}, \mathrm{CDCl}_{3}\right): \delta 142.0,140.9,140.0,132.5$, $131.4,130.0,129.6,128.7,127.2,127.0,126.5,126.0,125.7,125.7,124.3,123.5,123.3,122.8$, 120.4, 119.8, 119.8, 118.0, 114.3, 110.1, 109.9, 109.4, 102.3, 85.0, 43.6, 31.9, 29.5, 29.3, 29.1, 27.4, 22.7, 14.2. HRMS (ESI) calcd. for $\mathrm{C}_{48} \mathrm{H}_{40} \mathrm{~N}_{2}[\mathrm{M}+\mathrm{H}]^{+} 644.3191$; found 644.3187.

\section{3-((9,9-dioctylfluoren-2-yl)ethynyl)-9-octyl-6,9'-bicarbazole $\left(\mathrm{M} \equiv \mathrm{F}-\left(\mathrm{C}_{8} \mathrm{H}_{17}\right)_{2}\right)$}

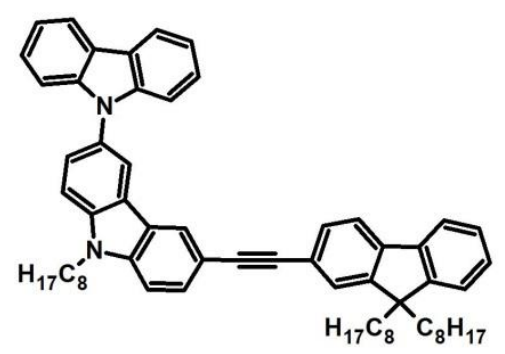

The crude product was purified by column chromatography (silica gel, hexane/dichloromethane, 10:1 $\rightarrow 5: 1 \mathrm{v} / \mathrm{v}$ ) and recrystallized from methanol, to give $\mathbf{M} \equiv \mathbf{F}$ $\left(\mathbf{C}_{8} \mathrm{H}_{17}\right)_{2}$ as a light brown solid (2.3 g, $77 \%$ yield). ${ }^{1} \mathbf{H} \mathbf{~ N M R}\left(400 \mathrm{MHz}, \mathrm{CDCl}_{3}\right): \delta 8.31(\mathrm{~s}, 1 \mathrm{H})$, $8.26(\mathrm{~s}, 1 \mathrm{H}), 8.20$ (d, $J=7.7 \mathrm{~Hz}, 2 \mathrm{H}), 7.73(\mathrm{~d}, J=8.5 \mathrm{~Hz}, 1 \mathrm{H}), 7.71-7.58(\mathrm{~m}, 4 \mathrm{H}), 7.56-7.50$ $(\mathrm{m}, 2 \mathrm{H}), 7.47(\mathrm{~d}, J=8.6 \mathrm{~Hz}, 1 \mathrm{H}), 7.44-7.39(\mathrm{~m}, 4 \mathrm{H}), 7.36-7.27(\mathrm{~m}, 5 \mathrm{H}), 4.40(\mathrm{t}, J=7.2 \mathrm{~Hz}$, $2 \mathrm{H}), 1.96(\mathrm{t}, J=8.0 \mathrm{~Hz}, 6 \mathrm{H}), 1.35-0.83(\mathrm{~m}, 33 \mathrm{H}), 0.80(\mathrm{t}, J=7.1 \mathrm{~Hz}, 6 \mathrm{H}), 0.71-0.52(\mathrm{~m}, 4 \mathrm{H})$. ${ }^{13}$ C NMR (101 MHz, $\left.\mathrm{CDCl}_{3}\right): \delta 151.03,150.77,141.85,141.07,140.72,140.57,139.90$, $130.44,129.91,129.44,127.36,126.84,125.89,125.85,125.60,124.77,124.26,123.46$, $123.18,122.88,122.59$, 121.94, 120.29, 119.91, 119.66, 119.60, 114.07, 109.97, 109.83, 109.16, 90.45, 89.07, 55.13, 43.58, 40.41, 31.82, 31.79, 30.06, 29.40, 29.24, 29.12, 27.39, 26.95, 23.75, 22.64, 22.60, 14.10, 14.07. HRMS (ESI) calcd. for $\mathrm{C}_{63} \mathrm{H}_{72} \mathrm{~N}_{2}[\mathrm{M}+\mathrm{H}]^{+} 856.5695$; found 856.5699 .

3-((4-methoxyphenyl)ethynyl)-9-octyl-6,9'-bicarbazole $\left(\mathrm{M} \equiv \mathrm{Ph}-\mathrm{OCH}_{3}\right)$

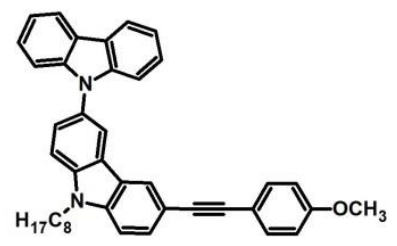


The crude product was purified by column chromatography (silica gel, hexane/dichloromethane, 10:1 $\rightarrow 5: 3 \mathrm{v} / \mathrm{v}$ ) and recrystallized from methanol, to give $\mathbf{M} \equiv \mathbf{P h}$ $\mathbf{O C H}_{3}$ as a light orange solid $(1.31 \mathrm{~g}, 65 \%$ yield $) .{ }^{1} \mathbf{H}$ NMR $\left(400 \mathrm{MHz}, \mathrm{CDCl}_{3}\right): \delta 8.26\left(\mathrm{dd}, J_{1}\right.$ $\left.=5.1 \mathrm{~Hz}, J_{2}=1.4 \mathrm{~Hz}, 2 \mathrm{H}\right), 8.20(\mathrm{~d}, J=7.7 \mathrm{~Hz}, 2 \mathrm{H}), 7.69\left(\mathrm{dd}, J_{l}=8.5 \mathrm{~Hz}, J_{2}=1.5 \mathrm{~Hz}, 1 \mathrm{H}\right)$, 7.65-7.57 (m, 2H), 7.53-7.48 (m, 2H), 7.46-7.39 (m, 5H), 7.31 (ddd, $J_{l}=7.9 \mathrm{~Hz}, J_{2}=5.6 \mathrm{~Hz}$, $\left.J_{3}=2.5 \mathrm{~Hz}, 2 \mathrm{H}\right), 6.92-6.85(\mathrm{~m}, 2 \mathrm{H}), 4.39(\mathrm{t}, J=7.2 \mathrm{~Hz}, 2 \mathrm{H}), 3.83(\mathrm{~s}, 3 \mathrm{H}), 2.07-1.88(\mathrm{~m}, 2 \mathrm{H})$, $1.51-1.22(\mathrm{~m}, 10 \mathrm{H}), 0.90(\mathrm{t}, J=6.7 \mathrm{~Hz}, 3 \mathrm{H}) .{ }^{13} \mathbf{C ~ N M R}\left(101 \mathrm{MHz}, \mathrm{CDCl}_{3}\right): \delta 159.38,141.88$, $140.60,139.89,132.90,129.83,129.37,125.88,125.56,124.07,123.46,123.17,122.54$, 120.28, 119.64, 119,61, 115.95, 114.24, 114.01, 109.93, 109.84, 109.11, 89.06, 87.78, 55.30, 43.55, 31.81, 29.39, 29.21, 29.09, 27.37, 22.63, 14.09. HRMS (ESI) calcd. for $\mathrm{C}_{63} \mathrm{H}_{72} \mathrm{~N}_{2}$ $[\mathrm{M}+\mathrm{H}]^{+}$574.2984; found 574.2985.

\section{3-((4-(trifluoromethyl)phenyl)ethynyl)-9-octyl-6,9'-bicarbazole $\left(\mathrm{M} \equiv \mathrm{Ph}-\mathrm{CF}_{3}\right)$}

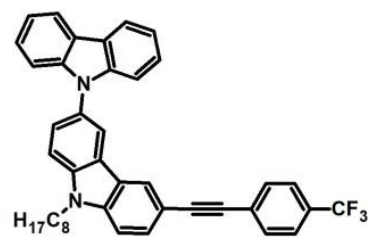

The crude product was purified by column chromatography (silica gel, hexane/dichloromethane, 10:1 $\rightarrow$ 5:2 v/v) and recrystallized from methanol, to give $\mathbf{M} \equiv \mathbf{P h}$ $\mathbf{C F}_{3}$ as a yellow solid (1.8 g, 84\% yield). ${ }^{1} \mathbf{H}$ NMR (400 MHz, $\left.\mathrm{CDCl}_{3}\right): \delta 8.30(\mathrm{~s}, 1 \mathrm{H}), 8.26$ (s, $1 \mathrm{H}), 8.20(\mathrm{~d}, J=7.7 \mathrm{~Hz}, 2 \mathrm{H}), 7.71(\mathrm{~d}, J=8.5 \mathrm{~Hz}, 1 \mathrm{H}), 7.68-7.57(\mathrm{~m}, 6 \mathrm{H}), 7.47(\mathrm{~d}, J=8.6 \mathrm{~Hz}$, 1H), 7.44-7.38 (m, 4H), 7.35-7.28 (m, 2H), 4.40 (t, $J=7.2 \mathrm{~Hz}, 2 \mathrm{H}), 2.06-1.89$ (m, 2H), 1.49$1.22(\mathrm{~m}, 10 \mathrm{H}), 0.90(\mathrm{t}, J=6.2 \mathrm{~Hz}, 3 \mathrm{H}) .{ }^{13} \mathbf{C} \mathbf{N M R}\left(101 \mathrm{MHz}, \mathrm{CDCl}_{3}\right): \delta 139.30,139.19,138.14$, 136.23, 136.04, 134.06, 132.78, 129.79, 128.75, 127.09, 126.00, 125.13, 124.88, 124.46, 123.82, 122.03, 121.45, 120.61, 120.18, 114.25, 111.02, 110.86, 107.08, 89.27, 85.58, 47.43, 31.65, 29.06, 28.96, 27.95, 27.55, 22.94, 14.02. HRMS (ESI) calcd. for $\mathrm{C}_{41} \mathrm{H}_{35} \mathrm{~F}_{3} \mathrm{~N}_{2}[\mathrm{M}+\mathrm{H}]^{+}$ 612.2752; found 612.2750 .

\section{Broadband dielectric spectroscopy (BDS)}

Dielectric measurements were performed using a Novocontrol GMBH Alfa impedance analyzer in the frequency range from $10^{-1}$ up to $10^{6} \mathrm{~Hz}$ at different temperatures corresponding to supercooled liquid state of investigated sizable systems. The temperature was precisely controlled by Quatro Cryosystem with high accuracy of $0.1 \mathrm{~K}$. The measurements were performed in a parallel plate steel capacitor $(20 \mathrm{~mm}$ diameter $)$ with a fixed distance between 
electrodes $(0.1 \mathrm{~mm})$ provided by fused silica fibers. Before measurements the samples were prepared by the melt quench cooling method. The capacitor plate with sample was heated up to the melting temperature and then was quenched by a fast transfer to a precooled cooper plate.

\section{X-ray diffraction (XRD)}

The X-ray diffraction measurements were performed at $293 \mathrm{~K}$ on a Rigaku-Denki D/MAX RAPID II-R diffractometer equipped with a rotating Ag anode, an incident beam (002) graphite monochromator, and an image plate in the Debye-Scherrer geometry. Samples were measured in glass capillaries with a diameter of $1.5 \mathrm{~mm}$. The background from an empty capillary was subtracted. The collected two-dimensional diffraction patterns were converted into one-dimensional intensity data versus the scattering vector, $Q=4 \pi(\sin \theta) / \lambda$, where $2 \theta$ is the scattering angle and the wavelength of the incident beam, $\lambda$, is equal to $0.56 \AA$. After a careful subtraction of the background and normalization, the diffraction patterns were fitted in the low- $Q$ range up to $1.75 \AA^{-1}$ by a superposition of three pseudo-Voigt functions. A contribution of incoherent Compton scattering was neglected because it is very week in this region. Also, a contribution from the intramolecular structure factor was neglected since it is very similar for all the studied compounds and gives only a weak scattering function in the low$Q$ range, as shown in Figure $\mathrm{S} 2$. The fits converged (the $R$-squared values were at least 0.995 ) and the values of main and pre-peak's positions, $Q_{M P}, Q_{P P 1}, Q_{P P 2}$, and full widths at the half peak maximum, fwhm $\mathrm{MP}_{\mathrm{P}}$, fwhm $\mathrm{PP} 1$, fwhmPP2, respectively, were obtained with uncertainties given by the fitting routine and listed in Table $\mathrm{S} 1$.

\section{Differential scanning calorimetry (DSC)}

Calorimetric measurements were carried out by Mettler-Toledo DSC 1 STARe system equipped with a HSS8 ceramic sensor (heat flux sensor with 120 thermocouples) and a liquid nitrogen cooling accessory. The devices were calibrated for temperature and enthalpy using indium and zinc standards. The samples were measured in aluminum crucibles with a $40 \mu \mathrm{L}$ volume. All DSC experiments were performed with a heating rate equal to $10 \mathrm{~K} / \mathrm{min}$ over temperature range from 225 to $500 \mathrm{~K}$ for $\mathrm{M} \equiv \mathrm{A}$, and 250 up to $450 \mathrm{~K}$ for $\mathrm{M} \equiv \mathrm{F}-\left(\mathrm{C}_{8} \mathrm{H}_{17}\right)_{2}$. Melting point was obtained as the onset of the peak, while the glass transition temperatures were determined as the midpoint of the heat capacity increment. 